\title{
Protective Effects of Statins Administration in European and North American Patients Infected with COVID-19: A Meta-Analysis
}

\author{
Diletta Onorato, MD ${ }^{1, *}$ Mairi Pucci, MD ${ }^{1, *}$ Giovanni Carpene, MD ${ }^{1}$ Brandon Michael Henry, MD \\ Fabian Sanchis-Gomar, MD,4 Giuseppe Lippi, MD ${ }^{1}$
}

${ }^{1}$ Section of Clinical Biochemistry, Department of Neurosciences, Biomedicine and Movement Sciences, University of Verona, Italy

${ }^{2}$ Cardiac Intensive Care Unit, The Heart Institute, Cincinnati Children's Hospital Medical Center, Ohio

${ }^{3}$ Department of Physiology, Faculty of Medicine, University of

Valencia, Valencia, Spain

${ }^{4}$ INCLIVA Biomedical Research Institute, Valencia, Spain

\begin{abstract}
Address for correspondence Diletta Onorato, MD, Section of Clinical Biochemistry, Department of Neurosciences, Biomedicine and Movement Sciences, P.le L.A Scuro, 10, 37134 Verona, Italy (e-mail: dilettaonorato@gmail.com).
\end{abstract}

Semin Thromb Hemost 2021;47:392-399.

\begin{abstract}
Keywords

- statins

- coronavirus disease

- COVID-19

- meta-analysis

Severe acute respiratory syndrome coronavirus 2 has spread rapidly throughout the world, becoming an overwhelming global health emergency. The array of injuries caused by this virus is broad and not limited to the respiratory system, but encompassing also extensive endothelial and systemic tissue damage. Since statins effectively improve endothelial function, these drugs may have beneficial effects in patients with coronavirus disease 2019 (COVID-19). Therefore, this investigation aimed to provide an updated overview on the interplay between statins and COVID-19, with particular focus on their potentially protective role against progression toward severe or critical illness and death. A systematic electronic search was performed in Scopus and PubMed up to present time. Data on statins use and COVID-19 outcomes especially in studies performed in Europe and North America were extracted and pooled. A total of seven studies met our inclusion criteria, totaling 2,398 patients (1,075 taking statins, i.e., 44.8\%). Overall, statin usage in Western patients hospitalized with COVID-19 was associated with nearly $40 \%$ lower odds of progressing toward severe illness or death (odds ratio: 0.59 ; $95 \%$ confidence interval: 0.35-0.99). After excluding studies in which statin therapy was started during hospital admission, the beneficial effect of these drugs was magnified (odds ratio: $0.51 ; 95 \%$ confidence interval: $0.41-0.64$ ). In conclusion, although randomized trials would be necessary to confirm these preliminary findings, current evidence would support a favorable effect of statins as adjuvant therapy in patients with COVID-19. Irrespective of these considerations, suspension of statin therapy seems highly unadvisable in COVID-19 patients.
\end{abstract}

The novel coronavirus disease 2019 (COVID-19) pandemic is sustained by severe acute respiratory syndrome coronaviru 2 (SARS-CoV-2). This is the third coronavirus outbreak that has occurred during the past 20 years, after

\footnotetext{
* Mairi Pucci and Diletta Onorato contributed equally to this work
}

those caused by SARS and Middle East respiratory syndrome (MERS) coronaviruses (CoVs). ${ }^{1,2}$ This new microorganism belongs to the CoV family, a class of enveloped, positive-sense, single-stranded RNA viruses, which typically cause respiratory, enteric, hepatic, and neurological diseases. ${ }^{3,4}$

(c) 2021. Thieme. All rights reserved. Thieme Medical Publishers, Inc., 333 Seventh Avenue, 18th Floor,

New York, NY 10001, USA
DOI https://doi.org/ 10.1055/s-0040-1722307. ISSN 0094-6176. and Preventing Thrombosis in COVID-19 -Part II; Guest Editors: Emmanuel J. Favaloro, PhD, FFSc (RCPA) and Giuseppe Lippi, MD. published online
January 22, 2021 
SARS-CoV-2 is transmitted principally through respiratory droplets, though contact/fomites and airborne (i.e., aerosol) transmission also plays a role in viral spread. ${ }^{5}$ The virus penetrates into the host cells by primarily binding to angiotensin-converting enzyme 2 (ACE2), and initially reproduces in the cells of lower and upper respiratory tracts. Viral invasion might then be followed by a dysregulated host inflammatory response in some genetically or clinically predisposed individuals, leading to life-threatening episodes of acute respiratory distress syndrome (ARDS) and/or multiple organ failure. ${ }^{6}$ ACE2 is a type I membrane protein expressed in lung, heart, kidney, and intestine and is mainly associated with cardiovascular disease. The protein consists of an $\mathrm{N}$-terminal peptidase domain $\mathrm{P}$ and a C-terminal Collectrin-like domain that ends with a single transmembrane helix and an approximately 40-residue intracellular segment. ${ }^{7}$ In addition to cleavage of angiotensin (Ang) I to produce Ang-(1-9) and cleavage of Ang II to produce Ang 1,7, ACE2 also provides a direct binding site for the spike (S) proteins of CoVs. Early studies have shown that SARS-CoV-2S protein binds to human ACE2 with a 10- to 20-fold higher affinity than SARS-CoV, thus explaining its higher virulence. ${ }^{8}$

The unfavorable progression of COVID-19, which can be seen in 5 to $15 \%$ of all patients with SARS-CoV-2 infections, is characterized by a dysregulated inflammatory reaction, which can also be accompanied by downregulation of ACE2 levels. ${ }^{9,10}$ On one hand this event may contribute to reduce the likelihood of being infected, on the other hand a lower ACE2 expression is associated with worse disease progression in those who have already been infected by SARS-CoV-2, as will be more specifically explained below. ${ }^{9,10}$ The adverse effects of COVID-19 were initially thought to be primarily limited to the respiratory tract (i.e., pneumonia and ARDS), but it is now clear that the virus can extend systematically, ${ }^{11}$ impacting a vast array of organs and tissues. The systemic propagation of the infection is frequently associated with development of a prothrombotic state, ${ }^{12}$ which manifests as micro- or macrovascular episodes of venous and/or arterial thrombosis, ${ }^{13,14}$ associated with unfavorable prognosis.

Recent data show that SARS-CoV-2 could directly infect the vascular endothelium and thereby impair endothelial cell function. Severe endothelial injuries associated with the presence of intracellular viruses, disrupted cell membranes, thrombosis with microangiopathy, and alveolar-capillary microthrombi have been described in COVID-19 patients. ${ }^{15,16}$ In accordance with these findings, it is now clear that patients with vascular comorbidities such as hypertension, cardiovascular disease, and even diabetes are at much greater risk of poor outcome. ${ }^{17-19}$

Although no universal pharmacological treatment against SARS-CoV-2 has been established to date, combinations of oxygenation, antiviral agents, immunosuppressive drugs, and anticoagulants, along with other compounds, are individually used. $^{20,21}$ Among the various agents investigated, recent evidence has emerged that statins (3-hydroxy-3-methylglutaryl coenzyme A -HMG-CoA- reductase inhibitors) may have a beneficial effect in preventing disease progression. ${ }^{22}$ Along with their lipid-lowering activity, statins are known to exert a kaleidoscope of pleiotropic effects on inflammation and oxidative stress, ${ }^{23}$ producing beneficial effects on cardiovascu- lar disease and thrombosis. ${ }^{24-26}$ Recent evidence has been provided that statins administration may be effective in upregulating ACE2, ${ }^{27}$ a mechanism that may lower the risk of developing severe ARDS in patients with SARS-CoV-2 infection, ${ }^{28}$ since this enzyme promotes the enzymatic conversion of AngII into Ang 1,7, which then counterbalances the proinflammatory and vasoconstrictive activity of the former peptide. ${ }^{29}$ Although the potential efficacy of antiatherosclerotic and antithrombotic mechanisms attributed to statins in COVID-19 requires further investigation, these drugs may represent a potentially promising therapy, at least as potential adjuvant drugs, especially due to their low cost, widespread availability, and relatively modest side effects. ${ }^{30,31}$

Thus, statins have been suggested as part of the arsenal to treat and/or attenuate COVID-19 symptoms and sequelae. ${ }^{23}$ Therefore, this work is aimed to provide an overview of recent scientific literature exploring the interplay between statins and outcome of COVID-19, especially in European and North American populations.

\section{Materials and Methods}

We performed an electronic search in Medline (PubMed interface) and Scopus, using the keywords "COVID-19" OR "SARSCoV-2" AND "statin," between 2019 and present time (i.e., September 28, 2020), restricting the search to articles published in English. The reference list of all documents was also reviewed to identify other potentially eligible studies. The title, abstract, and full text of the articles identified according to our search criteria were analyzed by two of the authors (D.O. and M.P.) and were considered eligible for inclusion in this literature review if they were case series (sample size $>10$ ) or observational studies reporting clear extractable data on the use of statins in laboratory-confirmed COVID-19 patients, and compared data regarding the use of statins between patients with severe/critical or nonsevere disease (or ARDS, or pulmonary embolism diagnosed by computer tomography pulmonary angiography) or between survivors and nonsurvivors. Severe/critical disease was defined as intensive care unit admission, need for mechanical ventilation, or ARDS, pulmonary embolism diagnosed by computer tomography pulmonary angiography, or death. We also excluded studies that only reported the generic term "lipidlowering agents" and no specific information on the class of drug along with studies in patients with specific pathologies that could lead to misinterpreting drug effect (i.e., patients with multiple myeloma). ${ }^{32}$ Reviews, case reports, and other editorial materials with no original data were also excluded. Disagreement arising during the selection assessment was resolved by discussion and consensus.

The data extracted from each article included authors, year of publication, title of the study, country, number of patients, age, number of patients taking statins or not, percentage of patients under statins use who developed severe disease or death versus those who did not, severity, infection criteria, and conclusions of the study (-Table $\mathbf{1}$ ).

Studies were selected in accordance with PRISMA (Preferred Reporting Items for Systematic Reviews and Metaanalyses) guidelines. ${ }^{33}$ 


\begin{tabular}{|c|c|c|c|c|c|c|c|c|c|c|c|c|c|c|}
\hline 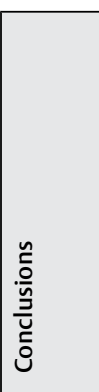 & 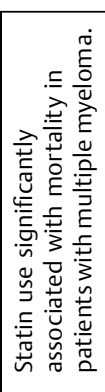 & 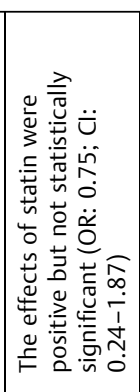 & 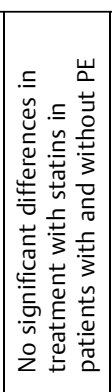 & 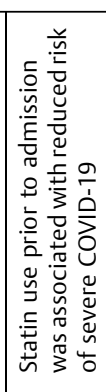 & 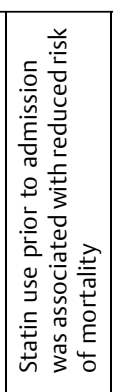 & 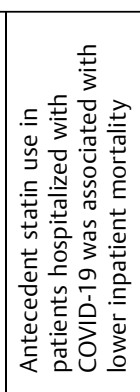 & 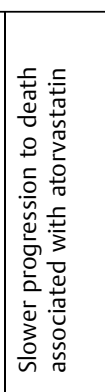 & 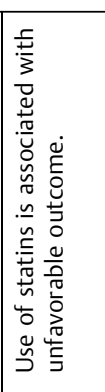 & 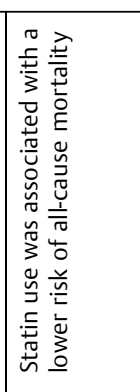 & 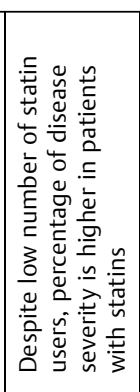 & $\Sigma$ & $\Sigma$ & $\Sigma$ & z \\
\hline 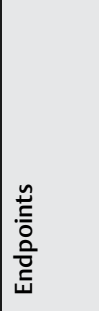 & 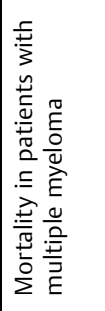 & 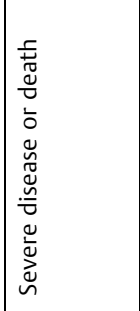 & 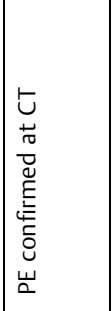 & 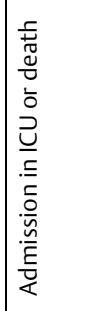 & \begin{tabular}{|l} 
营 \\
○े
\end{tabular} & \begin{tabular}{|l} 
䓌 \\
○ั
\end{tabular} & \begin{tabular}{|l} 
营 \\
口̆
\end{tabular} & 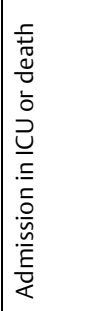 & 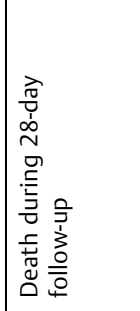 & 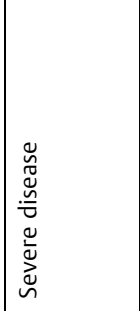 & 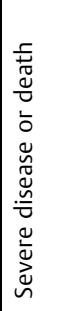 & 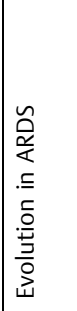 & 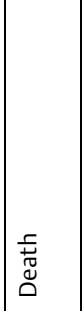 & \begin{tabular}{|l} 
䓌 \\
வ
\end{tabular} \\
\hline 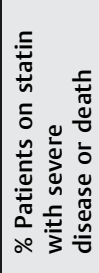 & 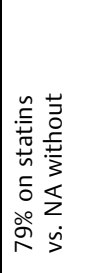 & 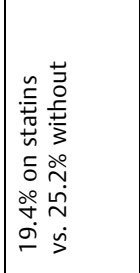 & 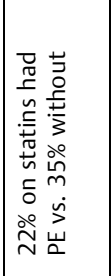 & 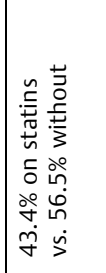 & 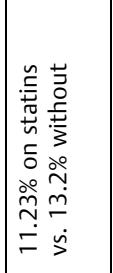 & 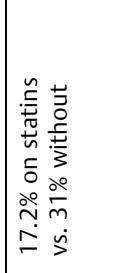 & 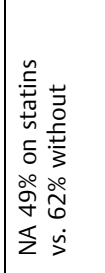 & 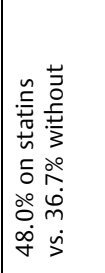 & 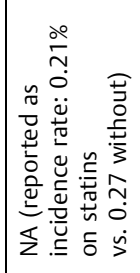 & 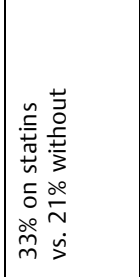 & s & 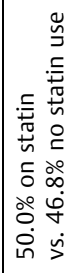 & 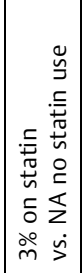 & $\Sigma$ \\
\hline & $\bar{m}$ & $\stackrel{\sim}{\simeq}$ & d & $\stackrel{\Xi}{\simeq}$ & 总 & $\stackrel{\infty}{0}$ & q & 8 & $\begin{array}{l}\tilde{L} \\
\stackrel{\sim}{\simeq}\end{array}$ & 莒 & $\ddot{\tilde{\vartheta}}$ & $\approx$ & Ø̆ & $\begin{array}{l}\text { ò } \\
\text { in } \\
m\end{array}$ \\
\hline 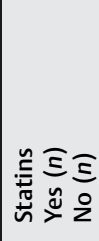 & $\grave{\sim}$ & $\bar{m}$ & $\grave{N}$ & g & $\infty$ & $\stackrel{\infty}{0}$ & 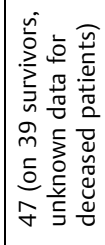 & $\underset{\infty}{\infty}$ & 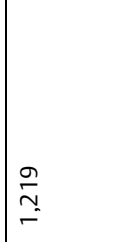 & $\stackrel{n}{\simeq}$ & $\underset{m}{\bar{d}}$ & 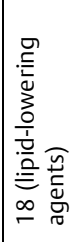 & $\stackrel{\infty}{m}$ & $\stackrel{\mathscr{F}}{\mathscr{F}}$ \\
\hline $\begin{array}{l}\text { त्र } \\
\text { 苂 }\end{array}$ & 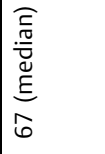 & $\begin{array}{l}n \\
\hat{q} \\
\vdots \\
r\end{array}$ & $\mid \begin{array}{l}n \\
\hat{1} \\
0 \\
n\end{array}$ & $\begin{array}{l}\infty \\
\hat{1} \\
\vdots \\
q\end{array}$ & $\mid \begin{array}{l}n \\
\hat{1} \\
i \\
\text { in }\end{array}$ & \begin{tabular}{|l}
$\bar{\alpha}$ \\
0 \\
0 \\
$\delta$
\end{tabular} & $\begin{array}{l}n \\
\hat{1} \\
\infty \\
n \\
n\end{array}$ & $\begin{array}{l}0 \\
0 \\
1 \\
i \\
n\end{array}$ & $\mid \begin{array}{l}\hat{1} \\
\dot{b} \\
\dot{\gamma}\end{array}$ & 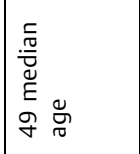 & $\begin{array}{l}n \\
\hat{1} \\
0 \\
i n \\
\text { in }\end{array}$ & $\begin{array}{l}0 \\
i \\
\infty \\
n \\
n\end{array}$ & $\begin{array}{l}t \\
\hat{1} \\
\dot{\sigma} \\
\dot{y}\end{array}$ & $\begin{array}{l}0 \\
0 \\
\hat{1} \\
\hat{n}\end{array}$ \\
\hline 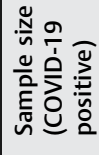 & $\hat{\sigma}$ & $\stackrel{\text { 号 }}{\sim}$ & $\bar{\sigma}$ & 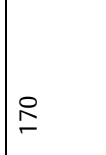 & 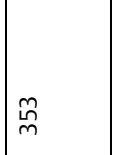 & 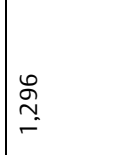 & $\infty$ & $\stackrel{\stackrel{丶}{\sim}}{ }$ & $\begin{array}{l}\bar{\alpha} \\
o \\
\tilde{m}\end{array}$ & $\stackrel{\infty}{n}$ & $\underset{-}{\stackrel{\circ}{-}}$ & 오 & $\stackrel{\bar{n}}{\tilde{o}}$ & $\begin{array}{l}\infty \\
\infty \\
\infty \\
m\end{array}$ \\
\hline 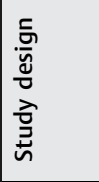 & 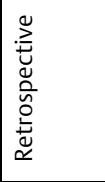 & 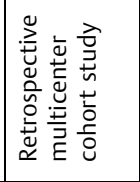 & 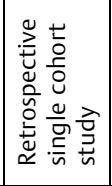 & 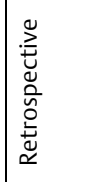 & 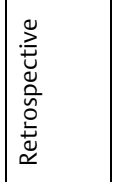 & 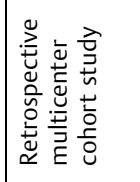 & 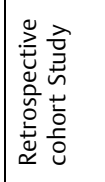 & 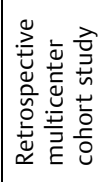 & 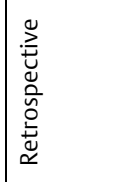 & 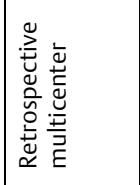 & $\begin{array}{l}\tilde{y} \\
\stackrel{\tilde{L}}{\tilde{u}} \\
\tilde{u} \\
\tilde{U}\end{array}$ & 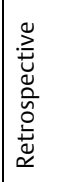 & 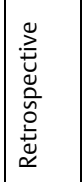 & 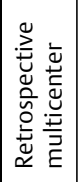 \\
\hline 袁 & 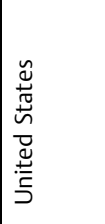 & 㝘 & $\begin{array}{l}\text { 壳 } \\
\tilde{\tilde{n}}\end{array}$ & 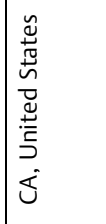 & 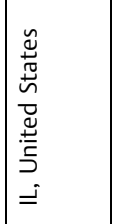 & 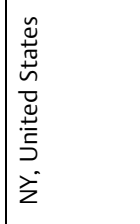 & 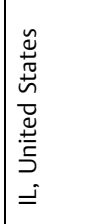 & 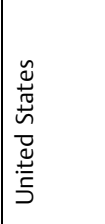 & 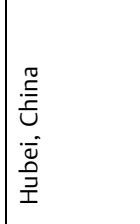 & 苞 & 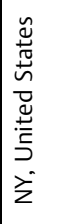 & 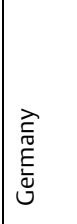 & 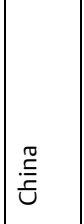 & 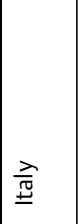 \\
\hline 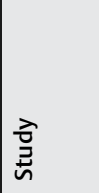 & 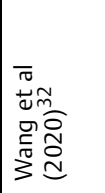 & 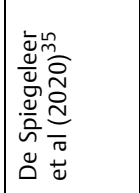 & 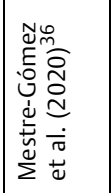 & 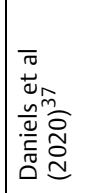 & 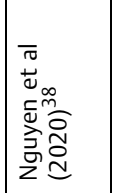 & 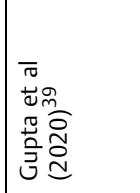 & 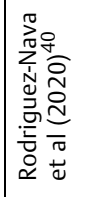 & 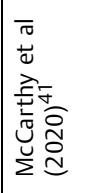 & 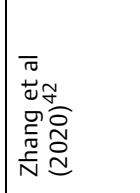 & 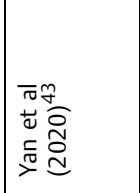 & 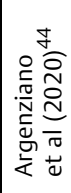 & 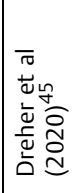 & 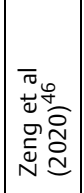 & 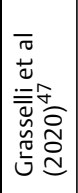 \\
\hline
\end{tabular}


For the purposes of pooling into a meta-analysis, Chinese studies (to limit ethnic and sample heterogeneity) and studies that did not provide useful data for statistical purposes were excluded from our analysis. A separate analysis of Chinese studies was unfeasible, as they lacked the essential data for statistical pooling. A composite endpoint of severe or fatal COVID-19 was employed for this analysis. The metaanalysis was finally performed with calculation of pooled odds ratio (OR) and 95\% confidence interval (95\% CI) using MetaXL, software Version 5.3 (EpiGear International Pty Ltd., Sunrise Beach, Australia). Heterogeneity among the included studies was probed employing both the chi-square $\left(X^{2}\right)$ test and the $I^{2}$ statistic. For the $\mathrm{X}^{2}$ test, significant heterogeneity among the studies was designated with a Cochran's $Q p$-value of $<0.10$. The $I^{2}$ statistic values were interpreted as 25,50 , and $75 \%$, indicating low, moderate, and high heterogeneity, respectively. ${ }^{34}$ To evaluate potential sources for heterogeneity among the studies, subgroup analyses by time of initiation of statin therapy (before or after hospital admission) and by administration of azithromycin (a macrolide drug with significant drug interaction with statins, especially fostering rhabdomyolysis) were performed.

\section{Results}

A total of 170 studies could be originally identified, 156 of which were finally excluded because they were duplicates or did not fulfill the above-mentioned eligibility criteria ( - Fig. 1).

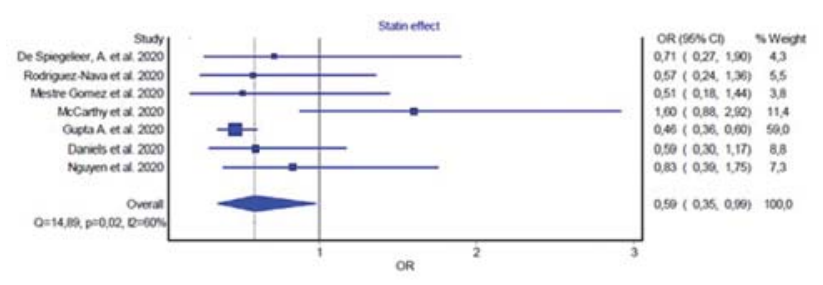

Fig. 2 Forest plot of the meta-analysis.

Overall, 14 studies met the inclusion criteria, ${ }^{32,35-47}$ and are summarized in - Table 1. After excluding studies from China or with inadequate data for pooling, the final meta-analysis was limited to seven retrospective studies performed in Western countries, ${ }^{35-41}$ totaling 2,398 patients, 1,075 of whom (44.8\%) were taking statins ( - Table $\mathbf{1}$ ).

Three of the included studies were based on patients with similar age range (50-76 years old), ${ }^{36,40,41}$ while the others included a mix of younger and older patients. Five out of seven studies evaluated patients with statin treatment before hospital admission, ${ }^{35-39}$ while the others considered treatments started before admission or during hospital stay. ${ }^{40,41}$ Notably, azithromycin ${ }^{48}$ was also administered to the vast majority of patients included in the study by McCarthy et al. ${ }^{41}$ Two studies applied death as the endpoint, two reported disease severity as the endpoint, and three reported both events.

The result of the meta-analysis of these seven studies performed in Western countries are reported in - Fig. 2, $^{35-41}$
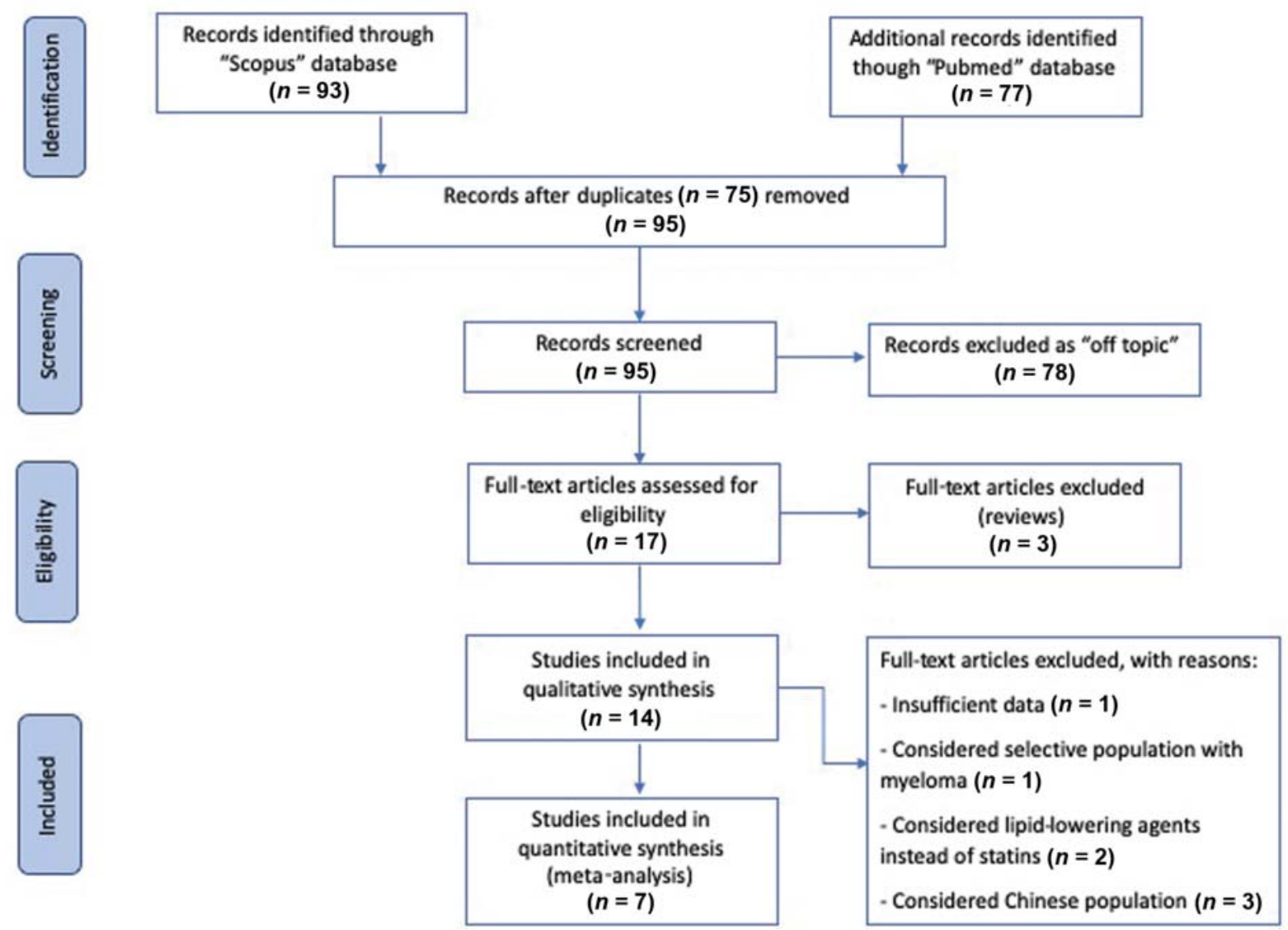

Fig. 1 PRISMA flow diagram of the literature search and selection process. 


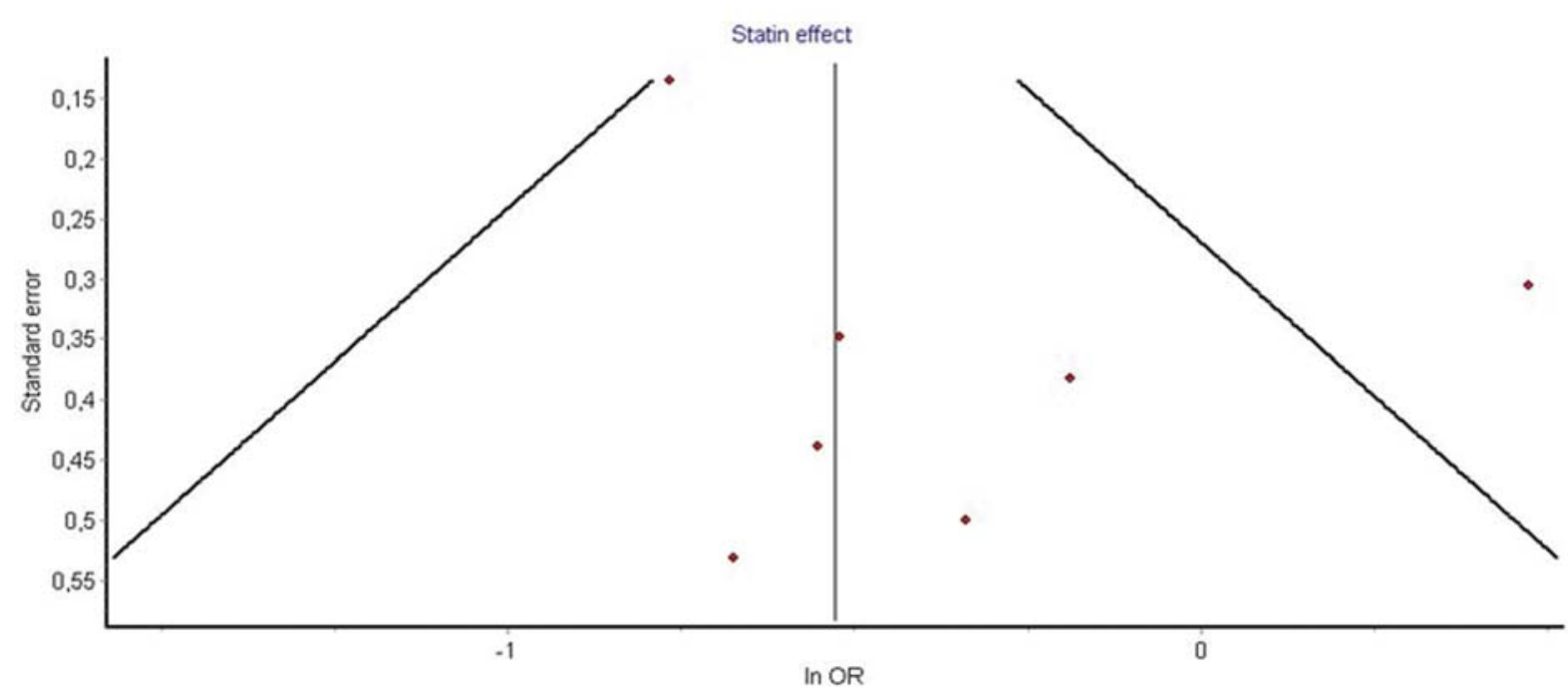

Fig. 3 Funnel plot of the meta-analysis: statins' protective effect versus lack of protection in hospitalized COVID-19 patients.

which demonstrate that COVID-19 patients taking statins had nearly $40 \%$ lower odds of progressing to the composite endpoint of severe/critical illness or death (OR: 0.59; $95 \% \mathrm{CI}$ : 0.35-0.99; Cochran's $Q p=0.02 ; I^{2}=60 \%$ ). Individually, six of these seven studies reported a reduced OR (i.e., $\mathrm{OR}<1$; protective effect of statins), ${ }^{35-40}$ while in only one study was the OR >1 (i.e., OR: 1.60; 95\% CI: 0.88-2.92). ${ }^{41}$ After excluding McCartney's study, in which patients were also given azithromycin, the beneficial effect of statins was magnified (OR: 0.52; 95\% CI: 0.42-0.64; Cochran's Q $\left.p=0.73, I^{2}=0 \%\right)$. The results did not substantially change after excluding both studies in which statin therapy was initiated after hospital admission (OR: 0.51; 95\% CI: 0.41-0.64; Cochran's Q $\left.p=0.61, I^{2}=0 \%\right) .{ }^{40,41}$ The funnel plot of all studies is shown in - Fig. 3, which demonstrated slight asymmetry.

\section{Discussion}

This article aimed to review the current scientific literature which has explored the potential impact of statins on clinical outcome of COVID-19 to date. Overall, the results of our analysis suggest a beneficial effect of these cardiovascular drugs in North American and European patients with SARSCoV-2 infection.

In fatal cases of human SARS-CoV-2 (as well as of SARS-CoV1 and MERS-CoV) infections, patients mostly progress to a severe form of acute respiratory distress, which requires mechanical ventilation and on autopsy demonstrates the classic histopathology findings of ARDS. ${ }^{49-51}$ ARDS is a life-threatening lung condition that causes diffuse alveolar damage in the lung, with hyaline membrane formation in alveoli in the acute stage, and subsequent interstitial widening, edema, and fibroblast proliferation in the organizing stage. ${ }^{52,53}$

Convincing evidence has been provided that release of inflammatory cytokines is strictly associated with development and progression of ARDS, thus confirming that the onset of a dysregulated inflammatory response is a primary event in promoting clinical deterioration in COVID-19 patients. ${ }^{54}$ The pathogenesis of COVID-19 encompasses hypercoagulability, inflammation, and decreased endothelial integrity, ${ }^{34}$ culminating in defective pulmonary vasoconstriction, shunting, and thrombotic microangiopathy. ${ }^{55-57}$ A potential beneficial effect of statin use thus possibly entails modulation (i.e., inhibition) of many underlying pathways, leading to potentially powerful anti-inflammatory and antithrombotic effects. In an animal model, atorvastatin was found to inhibit the expression of tolllike receptors, a family of sensor proteins which activate the myeloid differentiation primary response 88 pathway, thus underpinning its potential favorable effect in patients with COVID-19. ${ }^{58}$

Despite numerous investigations, it remains uncertain if statins could have a role in inhibiting the formation of thrombi in human subjects. To date, no study has demonstrated a substantial impact of statins in prevention of thrombosis. As such, statins are not clinically indicated for primary or secondary prevention of venous thromboembolism. ${ }^{59,60}$ Thus, the beneficial effects of statins in COVID-19 are more likely to be attributed to its anti-inflammatory properties (as well as improvement in endothelial function) than to any significant antithrombotic direct effect.

Nonetheless, another potential beneficial mechanism of HMG-CoA reductase inhibitors that needs to be mentioned in COVID-19 involves their effect on cholesterol reduction. During viral infections, viruses require host lipid metabolism for survival since lipids are implicated in membrane fusion, envelopment, and transformation. ${ }^{61}$ The host plasma membrane contains microdomains rich in proteins, sphingolipids, and cholesterol, named lipid rafts. An in vitro study investigated the role of these molecular structure in modulating the interaction between the spike protein of SARS-CoV-1 with ACE2 receptor, ${ }^{62}$ finding that cholesterol is required for efficient S-mediated binding to ACE2-containing cells and its depletion decreased the infectivity by approximately $50 \%$ in a single replication cycle. Given this evidence, it is possible that SARSCoV-2 would benefit from the presence of high cholesterol in the plasma membrane, which may hence facilitate its penetration into host cells. Thus, a statin-mediated inhibition of 
cholesterol biosynthesis pathway would result in decreased lipid raft formation and could hence be seen as a putative protective mechanism against SARS-CoV-2, as well as against other viral infections. . $^{63,64}$

Only one out of the seven studies included in our metaanalysis failed to report a favorable effect of statins in patients with SARS-CoV-2 infection. However, the concomitant administration of azithromycin in most patients enrolled in that study may have ultimately biased the outcome. In fact, muscle injury, possibly emerging from combined administration of the two drugs, may have contributed to attenuating the vascular benefits of statins, as clearly highlighted. ${ }^{41,65}$ As such, it is not surprising that the favorable outcome of statins in COVID-19 patients increased (i.e., from 40 to $\sim 50 \%$ lower risk of unfavorable progression, with narrower $95 \% \mathrm{CI}$ ) when this study was excluded from our meta-analysis. Unfortunately, no specific analysis could be performed to assess whether any single statin formulation would be more beneficial than another, since the type of drug administered was clearly specified in only one study (i.e., Atorvastatin $40 \mathrm{mg}$ ). ${ }^{39}$ Nonetheless, these findings support the start of new randomized controlled trials investigating statins as adjunctive therapy in COVID-19.

Our meta-analysis has some limitations. First, this pooled analysis had a relatively small sample size. Second, all studies were retrospective; two studies ${ }^{39,40}$ included patients in whom statin therapy was only started upon hospital admission, though their exclusion from the meta-analysis suggested an even larger favorable effect of statins. Finally, the use of a composite outcome likely introduced some heterogeneity into the analysis. However, subgroup analysis by study endpoint was unfeasible, as individual studies did not report data required to segregate patients by outcome, with the majority employing their own composite outcomes.

\section{Conclusion}

In conclusion, the results of this meta-analysis suggest a potential beneficial effect of statins in patients with COVID-19, especially when these drugs were initiated before hospital admission, but also lead the way to suggest that their administration could be beneficial for all patients hospitalized with COVID-19, as additional treatment for preventing unfavorable disease progression. Whether the favorable effect of statins in patients with SARS-CoV-2 infection may be direct (e.g., lowering lipids and decreasing the high cardiovascular risk characterizing COVID-19 patients), ${ }^{66}$ or is more predictably mediated by the pleiotropic effects of these drugs (i.e., anti-inflammatory, anti-thrombotic, anti-hypertensive), ${ }^{67}$ requires further investigation. Randomized controlled trials should be undertaken to explore the potentially diversified effects of statins as adjuvant therapy for improving outcomes in patients with SARS-CoV-2 infection. Irrespective of these considerations, it seems reasonable to conclude that statin therapy should at minimum not be suspended in patients with COVID-19.
Conflict of Interest

None declared.

\section{References}

1 Satija N, Lal SK. The molecular biology of SARS coronavirus. Ann N Y Acad Sci 2007;1102:26-38

2 Mackay IM, Arden KE. MERS coronavirus: diagnostics, epidemiology and transmission. Virol J 2015;12:222

3 Zhu N, Zhang D, Wang W, et al;China Novel Coronavirus Investigating and Research Team. A novel coronavirus from patients with pneumonia in China, 2019. N Engl J Med 2020;382(08):727-733

4 Weiss SR, Leibowitz JL. Coronavirus pathogenesis. Adv Virus Res 2011;81:85-164

5 Wiersinga WJ, Rhodes A, Cheng AC, Peacock SJ, Prescott HC. Pathophysiology, transmission, diagnosis, and treatment of coronavirus disease 2019 (COVID-19): a review. JAMA 2020;324(08): 782-793

6 Lippi G, Sanchis-Gomar F, Henry BM. COVID-19: unravelling the clinical progression of nature's virtually perfect biological weapon. Ann Transl Med 2020;8(11):693

7 Donoghue M, Hsieh F, Baronas E, et al. A novel angiotensinconverting enzyme-related carboxypeptidase (ACE2) converts angiotensin I to angiotensin 1-9. Circ Res 2000;87(05):E1-E9

8 Wrapp D, Wang N, Corbett KS, et al. Cryo-EM structure of the 2019-nCoV spike in the prefusion conformation. Science 2020; 367(6483):1260-1263

9 McGonagle D, Sharif K, O’Regan A, Bridgewood C. The role of cytokines including interleukin-6 in COVID-19 induced pneumonia and macrophage activation syndrome-like disease. Autoimmun Rev 2020;19(06):102537

10 Conti P, Ronconi G, Caraffa A, et al. Induction of pro-inflammatory cytokines (IL-1 and IL-6) and lung inflammation by Coronavirus19 (COVI-19 or SARS-CoV-2): anti-inflammatory strategies. J Biol Regul Homeost Agents 2020;34(02):327-331

11 Levi M, Thachil J. Coronavirus disease 2019 coagulopathy: disseminated intravascular coagulation and thrombotic microangiopathy-either, neither, or both. Semin Thromb Hemost 2020;46 (07):781-784

12 Henry BM, Vikse J, Benoit S, Favaloro EJ, Lippi G. Hyperinflammation and derangement of renin-angiotensin-aldosterone system in COVID-19: a novel hypothesis for clinically suspected hypercoagulopathy and microvascular immunothrombosis. Clin Chim Acta 2020;507:167-173

13 Di Minno A, Ambrosino P, Calcaterra I, Di Minno MND. COVID-19 and venous thromboembolism: a meta-analysis of literature studies. Semin Thromb Hemost 2020;46(07):763-771

14 Thachil J, Srivastava A. SARS-2 coronavirus-associated hemostatic lung abnormality in COVID-19: is it pulmonary thrombosis or pulmonary embolism? Semin Thromb Hemost 2020;46(07): 777-780

15 Ackermann M, Verleden SE, Kuehnel M, et al. Pulmonary vascular endothelialitis, thrombosis, and angiogenesis in Covid-19. N Engl J Med 2020;383(02):120-128

16 Calabrese F, Pezzuto F, Fortarezza F, et al. Pulmonary pathology and COVID-19: lessons from autopsy. The experience of European Pulmonary Pathologists. Virchows Arch 2020;477(03):359-372

17 Aggarwal G, Lippi G, Lavie CJ, Henry BM, Sanchis-Gomar F. Diabetes mellitus association with coronavirus disease 2019 (COVID-19) severity and mortality: a pooled analysis. J Diabetes 2020;12(11):851-855

18 Aggarwal G, Cheruiyot I, Aggarwal S, et al. Association of cardiovascular disease with coronavirus disease 2019 (COVID-19) severity: a meta-analysis. Curr Probl Cardiol 2020;45(08):100617

19 Lippi G, Wong J, Henry BM. Hypertension in patients with coronavirus disease 2019 (COVID-19):: a pooled analysis. Pol Arch Intern Med 2020;130:304-309 
20 Gil C, Ginex T, Maestro I, et al. COVID-19: drug targets and potential treatments. J Med Chem 2020;63(21):12359-12386

21 Yuan S, Chan CC, Chik KK, et al. Broad-spectrum host-based antivirals targeting the interferon and lipogenesis pathways as potential treatment options for the pandemic coronavirus disease 2019 (COVID-19). Viruses 2020;12(06):628

22 Sanchis-Gomar F, Perez-Quilis C, Favaloro EJ, Lippi G. Statins and other drugs: facing COVID-19 as a vascular disease. Pharmacol Res 2020;159:105033

23 Beltrán-García J, Osca-Verdegal R, Pallardó FV, et al. Oxidative stress and inflammation in COVID-19-associated sepsis: the potential role of anti-oxidant therapy in avoiding disease progression. Antioxidants 2020;9(10):E936

24 Zeiser R. Immune modulatory effects of statins. Immunology 2018;154(01):69-75

25 Yoon SJ, Yoon YW, Lee BK, et al. Potential role of HMG CoA reductase inhibitor on oxidative stress induced by advanced glycation endproducts in vascular smooth muscle cells of diabetic vasculopathy. Exp Mol Med 2009;41(11):802-811

26 Lim S, Sakuma I, Quon MJ, Koh KK. Differential metabolic actions of specific statins: clinical and therapeutic considerations. Antioxid Redox Signal 2014;20(08):1286-1299

27 Tikoo K, Patel G, Kumar S, et al. Tissue specific up regulation of ACE2 in rabbit model of atherosclerosis by atorvastatin: role of epigenetic histone modifications. Biochem Pharmacol 2015;93 (03):343-351

28 Wösten-van Asperen RM, Bos AP, Bem RA, et al. Imbalance between pulmonary angiotensin-converting enzyme and angiotensin-converting enzyme 2 activity in acute respiratory distress syndrome. Pediatr Crit Care Med 2013;14(09):e438-e441

29 Henry BM, Benoit S, Lippi G, Benoit J. Circulating plasma levels of angiotensin II and aldosterone in patients with coronavirus disease 2019 (COVID-19): a preliminary report. Prog Cardiovasc Dis 2020;63(05):702-703

30 Ju A, Hanson CS, Banks E, et al. Patient beliefs and attitudes to taking statins: systematic review of qualitative studies. Br J Gen Pract 2018;68(671):e408-e419

31 Zhao Z, Du S, Shen S, et al. Comparative efficacy and safety of lipidlowering agents in patients with hypercholesterolemia: a frequentist network meta-analysis. Medicine (Baltimore) 2019;98 (06): 14400

32 Wang B, Van Oekelen O, Mouhieddine TH, et al. A tertiary center experience of multiple myeloma patients with COVID-19: lessons learned and the path forward. J Hematol Oncol 2020;13 (01):94

33 Moher D, Liberati A, Tetzlaff J, Altman DGPRISMA Group. Preferred reporting items for systematic reviews and meta-analyses: the PRISMA statement. J Clin Epidemiol 2009;62(10):1006-1012

34 Higgins JP, Thompson SG, Deeks JJ, Altman DG. Measuring inconsistency in meta-analyses. BMJ 2003;327(7414):557-560

35 De Spiegeleer A, Bronselaer A, Teo JT, et al. The effects of ARBs, ACEis, and statins on clinical outcomes of COVID-19 infection among nursing home residents. J Am Med Dir Assoc 2020;21(07): 909.e2-914.e2

36 Mestre-Gómez B, Lorente-Ramos RM, Rogado J, et al;Infanta Leonor Thrombosis Research Group. Incidence of pulmonary embolism in non-critically ill COVID-19 patients. Predicting factors for a challenging diagnosis. J Thromb Thrombolysis 2020 (e-pub ahead of print). Doi: 10.1007/s11239-020-02190-9

37 Daniels LB, Sitapati AM, Zhang J, et al. Relation of statin use prior to admission to severity and recovery among COVID-19 inpatients. Am J Cardiol 2020;136:149-155

38 Nguyen AB, Upadhyay GA, Chung B, et al. Outcomes and cardiovascular comorbidities in a predominantly African-American population with COVID-19. medRxiv 2020. Doi: 10.1101/2020.06.28. 20141929

39 Gupta A, Madhavan MV, Poterucha TJ, et al. Association between antecedent statin use and decreased mortality in hospitalized patients with COVID-19. Res Sq 2020 (e-pub ahead of print). Doi: 10.21203/rs.21203.rs-56210/v21201

40 Rodriguez-Nava G, Trelles-Garcia DP, Yanez-Bello MA, Chung CW, Trelles-Garcia VP, Friedman HJ. Atorvastatin associated with decreased hazard for death in COVID-19 patients admitted to an ICU: a retrospective cohort study. Crit Care 2020;24(01):429

41 McCarthy CP, Murphy S, Jones-O'Connor M, et al. Early clinical and sociodemographic experience with patients hospitalized with COVID-19 at a large American healthcare system. EClinicalMedicine 2020;26:100504

42 Zhang XJ, Qin JJ, Cheng X, et al. In-hospital use of statins is associated with a reduced risk of mortality among individuals with COVID-19. Cell Metab 2020;32(02):176.e4-187.e4

43 Yan H, Valdes AM, Vijay A, et al. Role of drugs used for chronic disease management on susceptibility and severity of COVID-19: a large case-control study. Clin Pharmacol Ther 2020;108(06): 1185-1194

44 Argenziano MG, Bruce SL, Slater CL, et al. Characterization and clinical course of 1000 patients with coronavirus disease 2019 in New York: retrospective case series. BMJ 2020;369:m1996

45 Dreher M, Kersten A, Bickenbach J, et al. The characteristics of 50 hospitalized COVID-19 patients with and without ARDS. Dtsch Arztebl Int 2020;117(16):271-278

46 Zeng H, Zhang T, He X, et al. Impact of hypertension on progression and prognosis in patients with COVID-19: a retrospective cohort study in 1031 hospitalized cases in Wuhan, China. medRxiv 2020. Doi: 10.1101/2020.06.14.20125997

47 Grasselli G, Greco M, Zanella A, et al. Risk factors associated with mortality among patients with COVID-19 in intensive care units in Lombardy, Italy. JAMA Intern Med 2020;180(10):1345-1355

48 McMullan BJ, Mostaghim M. Prescribing azithromycin. Aust Prescr 2015;38(03):87-89

$49 \mathrm{Xu} \mathrm{Z}$, Shi L, Wang Y, et al. Pathological findings of COVID-19 associated with acute respiratory distress syndrome. Lancet Respir Med 2020;8(04):420-422

50 Ding Y, Wang $\mathrm{H}$, Shen $\mathrm{H}$, et al. The clinical pathology of severe acute respiratory syndrome (SARS): a report from China. J Pathol 2003;200(03):282-289

$51 \mathrm{Ng}$ DL, Al Hosani F, Keating MK, et al. Clinicopathologic, immunohistochemical, and ultrastructural findings of a fatal case of Middle East respiratory syndrome coronavirus infection in the United Arab Emirates, April 2014. Am J Pathol 2016;186(03): 652-658

52 Gibson PG, Qin L, Puah SH. COVID-19 acute respiratory distress syndrome (ARDS): clinical features and differences from typical pre-COVID-19 ARDS. Med J Aust 2020;213(02):54.e1-56.e1

53 Thompson BT, Chambers RC, Liu KD. Acute respiratory distress syndrome. N Engl J Med 2017;377(06):562-572

54 Wu L, O'Kane AM, Peng H, Bi Y, Motriuk-Smith D, Ren J. SARS-CoV-2 and cardiovascular complications: from molecular mechanisms to pharmaceutical management. Biochem Pharmacol 2020;178:114114

55 Fogarty H, Townsend L, Ni Cheallaigh C, et al. More on COVID-19 coagulopathy in Caucasian patients. Br J Haematol 2020;189(06): 1060-1061

56 Gavriilaki E, Brodsky RA. Severe COVID-19 infection and thrombotic microangiopathy: success does not come easily. Br J Haematol 2020;189(06):e227-e230

57 Tang N, Li D, Wang X, Sun Z. Abnormal coagulation parameters are associated with poor prognosis in patients with novel coronavirus pneumonia. J Thromb Haemost 2020;18(04):844-847

58 Karalis DG. Are statins safe in patients with COVID-19? J Clin Lipidol 2020;14(04):396-397

59 Watanabe T, Oku K, Amengual O, et al. Effects of statins on thrombosis development in patients with systemic lupus erythematosus and antiphospholipid antibodies. Lupus 2018;27(02):225-234

60 Gaertner S, Cordeanu EM, Nouri S, Mirea C, Stephan D. Statins and prevention of venous thromboembolism: myth or reality? Arch Cardiovasc Dis 2016;109(03):216-222 
61 Lorizate M, Kräusslich HG. Role of lipids in virus replication. Cold Spring Harb Perspect Biol 2011;3(10):a004820

62 Glende J, Schwegmann-Wessels C, Al-Falah M, et al. Importance of cholesterol-rich membrane microdomains in the interaction of the $S$ protein of SARS-coronavirus with the cellular receptor angiotensin-converting enzyme 2. Virology 2008;381(02):215-221

63 Rodrigues-Diez RR, Tejera-Muñoz A, Marquez-Exposito L, et al. Statins: could an old friend help in the fight against COVID-19? $\mathrm{Br}$ J Pharmacol 2020;177(21):4873-4886

64 Abu-Farha M, Thanaraj TA, Qaddoumi MG, Hashem A, Abubaker J, Al-Mulla F. The role of lipid metabolism in COVID-19 virus infection and as a drug target. Int J Mol Sci 2020;21(10):3544
65 Strandell J, Bate A, Hägg S, Edwards IR. Rhabdomyolysis a result of azithromycin and statins: an unrecognized interaction. Br J Clin Pharmacol 2009;68(03):427-434

66 Wang G, Zhang $Q$, Zhao $X$, et al. Low high-density lipoprotein level is correlated with the severity of COVID-19 patients: an observational study. Lipids Health Dis 2020;19 (01):204

67 Antonopoulos AS, Margaritis M, Lee R, Channon K, Antoniades C. Statins as anti-inflammatory agents in atherogenesis: molecular mechanisms and lessons from the recent clinical trials. Curr Pharm Des 2012;18(11):1519-1530 